\title{
Progress in Cryo-FIB Preparation of Biological Specimens for Cryo-TEM
}

\author{
C. Hsieh ${ }^{1}$, T. Wagenknecht ${ }^{1}$, and M. Marko ${ }^{1,2}$
}

\begin{abstract}
1. New York State Department of Health, Wadsworth Center, Empire State Plaza, Albany, NY 12201USA
2. College of Nanoscale Science and Engineering of the University at Albany, 251 Fuller Rd., Albany, NY 12203 USA
\end{abstract}

Since our introduction of the technique [1,2], the benefits of using cryo-FIB milling to thin vitreously frozen biological material for cryo-TEM observation have been confirmed by the subsequent development of the technique by several other laboratories $[3,4,5,6]$.

The impetus for our cryo-FIB development is to carry out high-resolution cryo-TEM tomography on frozen-hydrated biological specimens. Our ultimate purpose is to identify and reconstruct macromolecular complexes in-situ, comparing their 3-D structures to those obtained from high-resolution single-particle reconstruction of the isolated complexes, and identifying associated structures in their native environment [7; Fig. 1].

In the case of thick specimens, an alternative to cryo-FIB milling, cryo-ultramicrotomy, suffers from artifacts due to the mechanical cutting action of the knife, and these artifacts are avoided with ion-beam milling [2]. The most bothersome artifact of cryo-ultramicrotomy, with respect to subtomogram averaging of in-situ macromolecular complexes, is compression. Since all cellular components do not compress to the same degree, computational correction is impractical.

There are four main issues in the improvement of FIB-based cryo-preparation: (1) locating a particular object of interest, (2) extending the technique to the full range of specimen types, (3) better avoidance of frost during cryo-transfer steps, and (4) improving specimen-handling for greater convenience and to avoid specimen loss or damage.

The use of correlative cryo light/electron microscopy to locate cells of interest for cryo-FIB milling and subsequent cryo-TEM represents important progress [3]. We expect that advances in cryo-light microscopy will lead to better localization of sub-cellular features, and that the use of the technique can be extended to thick, frozen-hydrated tissue samples. Other ways to locate objects of interest in frozen samples are also under consideration.

Most of this cryo-FIB work has been done with cells that have been plunge-frozen on TEM grids [2,3,5]. However since many cells may be as thick as $20 \mu \mathrm{m}$, high-pressure freezing (HPF) may be the only way to adequately freeze them for high-resolution TEM. Cell suspensions in copper tubes have been prepared by HPF for subsequent cryo-FIB milling [4]. Wishing to make the cryo-FIB method "universal", even in our earliest work [8] we began to use custom-designed HPF specimen carriers that make it possible to prepare any type of cell or tissue for cryo-FIB milling and subsequent cryo-TEM [9]. Whether frozen in copper tubes or in custom carriers, the HPF material is pre-trimmed using a cryo-ultramicrotome, to speed up the subsequent ion-beam milling step.

Like all practitioners of the technology, we have been designing means to cover the specimen for protection from frost as it is transferred from the cryo-FIB to the cryo-TEM. While no method so far completely eliminates frost, most labs produce specimens with sufficiently little frost. Nevertheless, it is worthwhile to continue development in an effort to further reduce frost, which becomes especially important when aiming to study a specific organelle that might be sparse in the cell. 
Our current development work aims to provide the fixtures, tools and workflow needed to conveniently and reliably produce cryo-FIB-milled specimens -- of any biological origin -- for cryoTEM imaging and tomographic tilt-series collection. This work centers on a device to carry the specimen (which may be a TEM grid or a special HPF carrier) in an easy-to-handle holder, which in turn can be mounted in the cryo-microtome for trimming, then on the FIB coldstage (e.g. our Zeiss Neon-40 EsB FIB/SEM), and finally in the cryo-TEM (e.g. our JEOL JEM-4000FX). Our hardware also includes the most-recent shuttered cryostage specimen block for the Leica Microsystems VCT$100 \mathrm{FIB} / \mathrm{SEM}$ cryotransfer system (which we slightly modified), a modified cryo-transfer holder for the TEM (based on a Gatan model 626), and special tools and fixtures to improve ease of handling.

\section{References}

[1] M. Marko et al., J. Microsc. 222 (2006) 42.

[2] M. Marko et al., Nat. Meth. 4 (2007) 215.

[3] A. Rigort et al., J. Struct. Biol. 172 (2010) 169.

[4] M. Hayles et al., J. Struct. Biol. 172 (2010) 180.

[5] K. Strunk et al., J. Microsc. 247 (2012) 220.

[6] S. Rubino et al. J. Struct. Biol. 180 (2012) 572.

[7] C. Renken et al. J. Struct. Biol. 165 (2009) 53.

[8] M. Marko et al. Microsc. Microanal. 12(2) (2006) 98.

[9] M. Marko et al., Microsc. Microanal. 16(2) (2010) 1082.

[10] Supported by NIH grants GM097010 (M. Marko) and AR40615 (T. Wagenknecht).
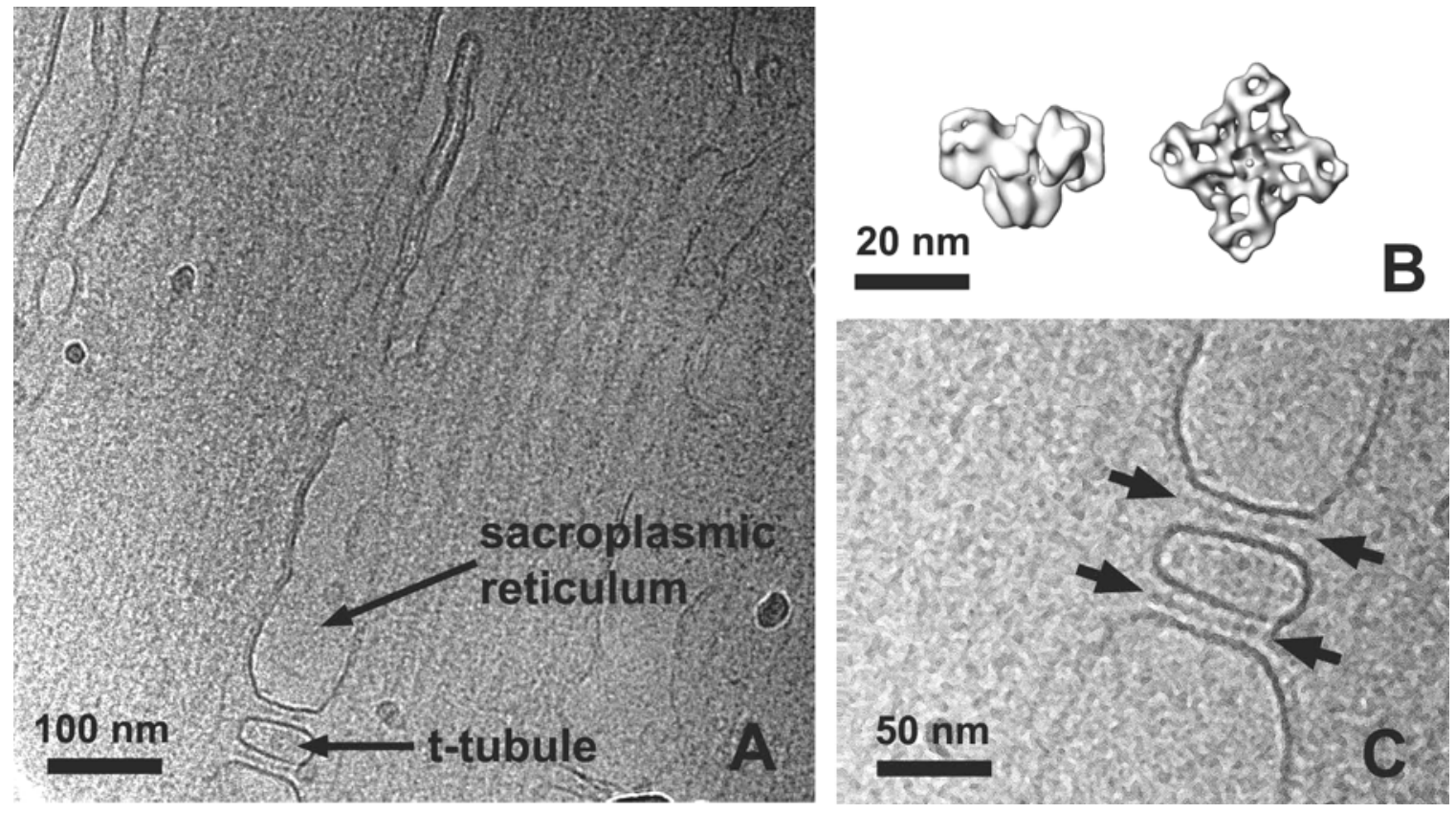

FIG. 1. Cryo-TEM images of 150-nm-thick FIB-thinned HPF skeletal muscle tissue recorded at 400kV. (A) Overview. (B) Side and top views of the ryanodine receptor from single-particle reconstruction. (C) Enlargement of the lower part of (A), ryanodine receptors indicated by the black arrows. In this view, two RyRs lie next to each other in side view, on both sides of the t-tubule, with the tip of the pyramid embedded in the membrane of the sarcoplasmic reticulum. 